1 The effect of COVID-19 vaccination in Italy and perspectives for 2 "living with the virus"

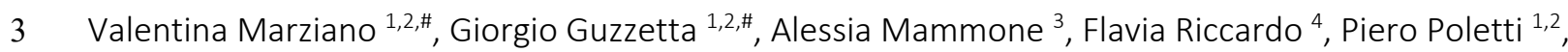
4 Filippo Trentini ${ }^{1,2}$, Mattia Manica ${ }^{1,2}$, Andrea Siddu ${ }^{3}$, Antonino Bella ${ }^{4}$, Paola Stefanelli ${ }^{4}$, Patrizio

5 Pezzotti ${ }^{4}$, Marco Ajelli ${ }^{5,6, \wedge}$, Silvio Brusaferro ${ }^{4, \wedge}$, Giovanni Rezza ${ }^{3, \wedge}$, Stefano Merler ${ }^{1,2, \wedge, *}$

6 Affiliations:

71 Center for Health Emergencies, Bruno Kessler Foundation, Trento, Italy

82 Epilab-JRU, FEM-FBK Joint Research Unit, Trento, Italy

93 Health Prevention, Ministry of Health, Rome, Italy

104 Istituto Superiore di Sanità, Rome, Italy

115 Laboratory for Computational Epidemiology and Public Health, Indiana University School of Public

12 Health, Bloomington, United States

136 Laboratory for the Modeling of Biological and Socio-technical Systems, Northeastern University,

14 Boston, United States

15 \# joint first authors

$16 \wedge$ joint senior authors

17 * corresponding author: Stefano Merler, merler@fbk.eu

\title{
18 Abstract
}

19 Vaccination campaigns against COVID-19 are allowing the progressive release of physical distancing restrictions in many countries. However, the global spread of the highly transmissible Delta variant has likely suppressed the residual chances of SARS-CoV-2 elimination through herd immunity alone. Here we assess the impact of the vaccination program in Italy since its start on December 27, 2020 and evaluate possible prospects for reopening the society while at the same time keeping COVID-19 under control. To this aim, we propose a mathematical modeling framework where levels of social activity are adjusted to match the time-series of the net reproduction number as estimated from surveillance data. We compared the estimated level of social contacts, number of deaths, and transmission potential with those of a counterfactual scenario where the same epidemic trajectory is obtained in absence of vaccination. We then evaluate the prospective impact of different scenarios of vaccination coverage and different social activity levels on SARS-CoV-2 reproduction number. We estimate that by June 30, 2021, the COVID-19 vaccination program allowed the resumption of about half the social contacts that were recorded in pre-pandemic times; in absence of vaccination, only about one third could have been resumed to obtain the same number of cases, with the added cost of about 12,100 (95\% Cl: 6,600-21,000) extra deaths (+27\%; $95 \% \mathrm{Cl}: 15-47 \%)$ between December 27 , 2020 and June 30, 2021. We show that the negative effect of the Delta variant diffusion in July was entirely offset by vaccination in the month of July and August 2021. Finally, we estimate that a complete return to the pre-pandemic life could be safely attained only if $>90 \%$, including children from 5 years on, will be vaccinated using mRNA vaccines developed in 2020. In any case, increasing the vaccination coverage will allow further margins for societal reopening even in absence of a pediatric vaccine. These results may support the definition of vaccination targets for countries that have already achieved a broad population coverage.

NOTE: This preprint reports new research that has not been certified by peer review and should not be used to guide clinical practice. 
medRxiv preprint doi: https://doi.org/10.1101/2021.03.19.21253893; this version posted November 15,2021 . The copyright holder for this preprint (which was not certified by peer review) is the author/funder, who has granted medRxiv a license to display the preprint in

It is made available under a CC-BY-NC-ND 4.0 International license .

\section{Introduction}

Since December 2020, vaccination against COVID-19 is being rolled out in all countries of the world, in a race to put an end to the devastating effects of the pandemic in terms of lives lost [1], hospital congestion [2], economic disruption [3], and mental health [4]. While African countries are painfully struggling to have access to vaccines and to distribute them (only $4.5 \%$ of the population in Africa is fully vaccinated, as of October 1, 2021 [5]), most high-income countries had a fast deployment, with over half of their citizens being fully immunized by July 2021 [5]. Thanks to the high efficacy and effectiveness of the licensed vaccines against SARS-CoV-2 infection, severe disease, and death [6-10], and to the prioritization of the highest risk categories, these countries were able to limit the damages caused by the emergence of the hypertransmissible Delta variant [11-13]. In the European Union, for example, despite ample relaxations of physical distancing restrictions conceded by governments over the summer of 2021, the peak mortality never exceeded 1.5 deaths per million since Delta become dominant in July, as compared to values over three times higher from November 2020 through April 2021 [5]. Similarly, the incidence of confirmed cases remained within about 150 cases per million, a value that is lower than those systematically observed between mid-October 2020 and mid-May 2021 [5]. With the ongoing progress of immunization campaigns, there is a need to quantitatively assess their impact on health and social activities, as well as to evaluate potential future epidemiological scenarios. In particular, as the emergence of the Delta variant has severely dwindled chances to eliminate SARS-CoV-2 [14] in countries that have not managed to maintain a zero-COVID approach [15], there is a need to identify strategic objectives towards "living with COVID-19" [16] at least in the medium term.

In this study, we use a mathematical model of SARS-CoV-2 transmission, informed by detailed realworld data, to retrospectively evaluate the effect of COVID-19 vaccination in Italy during the first half of 2021, and to prospectively assess potential future scenarios associated to different coverage levels.

\section{Results}

We adapted an age-structured, compartmental model of SARS-CoV-2 transmission in Italy $[17,18]$ that estimates the level of social activity needed to match the net reproduction number, as computed from official epidemic curves recorded in the national integrated surveillance system $[10,19]$. The level of social activity is expressed in terms of the proportion of social contacts measured before the pandemic [20]. The model keeps into account the dynamics of age-specific population immunity due to both infection [17], the progression of the vaccination campaign [21], and waning of immunity. We assume that protection from both natural and vaccine-induced immune response wanes exponentially with a baseline average duration of 2 years [22, 23]. We assume that successfully vaccinated individuals are not fully immune ("leaky vaccine") with different efficacy values for preventing infection and lethal disease. We tuned the model with data from the initial phase of the vaccination campaign (December 27 - June 30, 2021), when the SARS-CoV-2 Alpha variant was dominant in the country [24], and we project model results for the future by considering the progression of the vaccination campaign and the dominance of the Delta variant as of October 2021 $[25,26]$. Further details on the model are provided in Section Methods.

Retrospective analysis. The model reproduces the observed number of COVID-19 cases and deaths in vaccinated (partially or fully) and unvaccinated individuals over the first half of 2021 (Figure 1A-C). 
medRxiv preprint doi: https://doi.org/10.1101/2021.03.19.21253893; this version posted November 15,2021 . The copyright holder for this preprint (which was not certified by peer review) is the author/funder, who has granted medRxiv a license to display the preprint in

It is made available under a CC-BY-NC-ND 4.0 International license .

Considering the population immunity acquired from both vaccination and infection, a significant fraction of the Italian population $(36.2 \%, 95 \% \mathrm{Cl}: 35.9-36.7 \%)$ was estimated to be fully susceptible to SARS-CoV-2 as of June 30, 2021, with high heterogeneity by age (Figure 1D). This population immunity profile would have been insufficient to avoid potential successive outbreaks if caution was not applied when lifting physical distancing restrictions; for example, a complete resumption of pre-pandemic social activity would result in an effective reproduction number of $1.9(95 \% \mathrm{Cl}: 1.8-2.1)$ on June 30, 2021, even in absence of the more transmissible Delta variant.

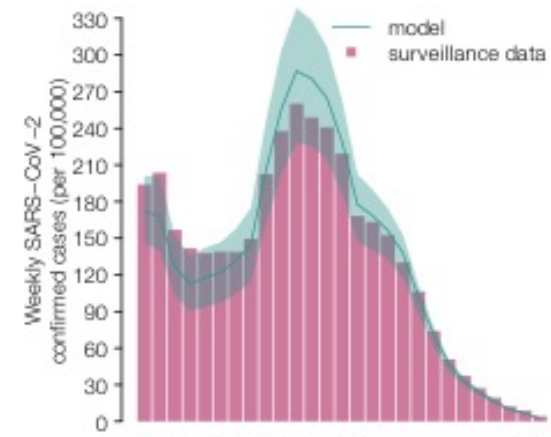

A.
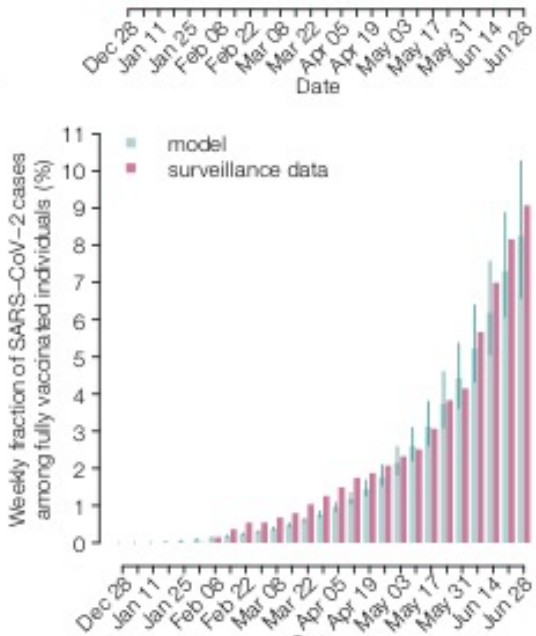

C. Date

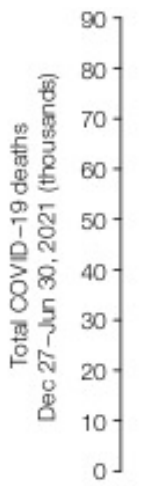

B.

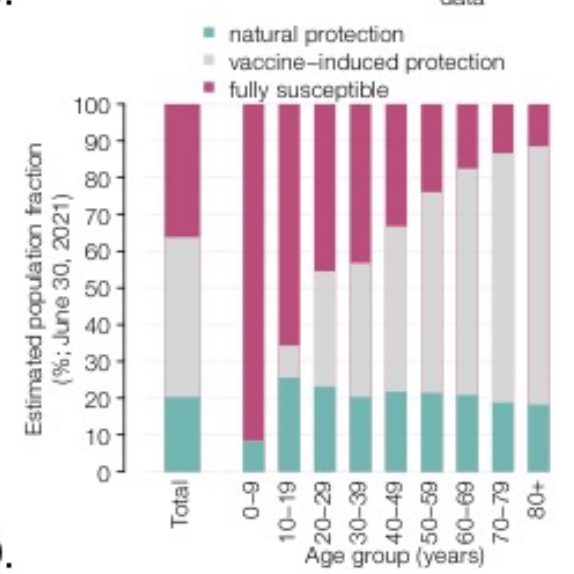

Figure 1. Characteristics of the COVID-19 epidemic in Italy during the first half of 2021. A) Weekly incidence per 100,000 population of COVID-19 confirmed cases (the $x$ axis reports the starting day of the considered week); gray bars: data from the Italian Integrated Surveillance System [27]; green lines: mean of the model estimates; green shaded area: $95 \% \mathrm{Cl}$. B) Total number of COVID-19 deaths over the study period (in thousands) among vaccinated (partially or fully) and unvaccinated individuals; green: model; red: data from the Italian Integrated Surveillance System [28]. C) Weekly percentage of confirmed COVID-19 cases occurring in fully vaccinated individuals over the total. The fraction of cases in completely vaccinated individuals increases over time because of the progressive increase in the vaccinated population. Bars: mean model estimate and observed data; vertical lines: $95 \% \mathrm{Cl}$. D) Estimated immunity profile of the Italian population, overall and by age groups, on June 30, 2021. Individuals who have been infected after being vaccinated or who have been vaccinated despite still having a protection from infection are counted under the natural protection bar; individuals who have never been infected or who have lost their natural protection and were vaccinated (partially or fully) are included under the vaccine-induced protection bar; individuals who were never vaccinated nor infected, or who were infected but lost their natural protection, or who were vaccinated but lost their vaccine-induced protection are included under the fully susceptible bar.

To evaluate the impact of the COVID-19 vaccination program in Italy over the first half of 2021, we simulated a scenario where we assume that the actual epidemic trajectory would be maintained, in 
medRxiv preprint doi: https://doi.org/10.1101/2021.03.19.21253893; this version posted November 15,2021 . The copyright holder for this preprint (which was not certified by peer review) is the author/funder, who has granted medRxiv a license to display the preprint in

It is made available under a CC-BY-NC-ND 4.0 International license .

109

110

111

112

113

114

115

116

117

118

119

120

121

122

123

124

125

126

127

128

129

130

131

132

133

134

135 absence of vaccination, by an appropriate reduction in social activity over time, due to both governmental measures and individual behavioral choices. Under these hypotheses, a decrease of about one fourth - from 48\% (95\% Cl: 44-51\%) to 35\% (95\% Cl: 33-37\%) - of the average proportion of active social contacts at the end of June 2021 would have been needed (Figure 2A). Furthermore, we estimate that about 12,100 additional deaths $(95 \% \mathrm{Cl}: 6,600-21,000$, corresponding to an increase of $27 \%, 95 \% \mathrm{Cl}: 15-47 \%$ ), would have occurred in the population even under the same cumulative number of cases (Figure 2B), mostly because of a larger proportion of infections among the high-risk segments of the population. Finally, we estimate that the potential for successive waves would be much larger due to the lower population immunity under this scenario, with an estimated effective reproduction number of 2.6 (95\% Cl: 2.4-2.8) (Figure $2 \mathrm{C}$ ).

If the Alpha variant had remained dominant until September 2020, we estimated that the progress of the vaccination campaign in July and August 2021 (Figure 3A) would have resulted in a decline of the reproduction number from 0.92 ( $95 \% \mathrm{Cl}$ : 0.88-0.95) on June 30 to 0.61 (95\% Cl: 0.54-0.71) on September 7, 2021 (Figure 3B). However, the Delta variant had rapidly replaced Alpha in July 2021 [25]. Considering a 50\% increase in transmissibility of the Delta variant [11-13], the estimated reproduction number on September 7, 2021, is 0.91 ( $95 \% \mathrm{Cl}: 0.81-1.06)$, close to the observed value of 0.83 (95\% Cl: 0.82-0.84) [29]. Thus, the increased transmissibility of the new variant and the progress of the vaccination campaign in the summer of 2021 have essentially leveled out, resulting in similar values of the reproduction number at the end of June and beginning of September, 2021.
A.

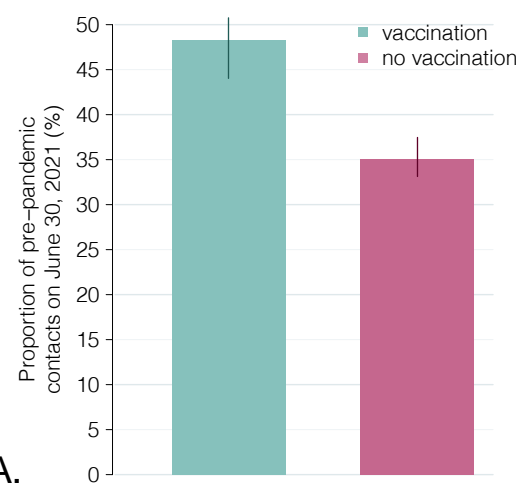

B.
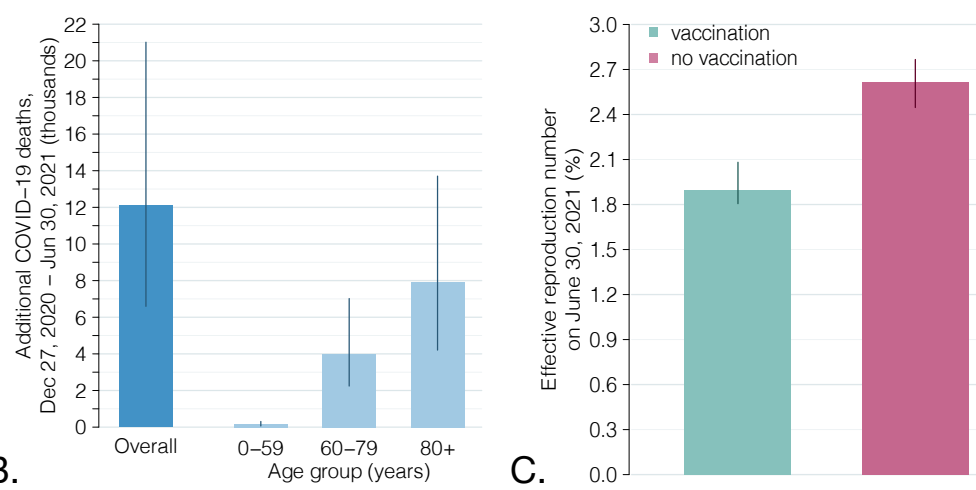

Figure 2. Impact of the vaccination program during the first half of 2021. A) Estimated active social contacts on June 30, 2021, as a proportion of pre-pandemic contacts, with and without a vaccination program, under the constraint that the two scenarios reproduce the same observed epidemic trajectory. B) Estimated additional COVID-19 deaths between December 27, 2020 and June 30, 2021, total and by age group, under a novaccination scenario. C) Effective reproduction number (i.e., under complete resumption of pre-pandemic contacts) on June 30, 2021, with and without vaccination. Bars: mean estimates; vertical lines: $95 \% \mathrm{Cl}$. 


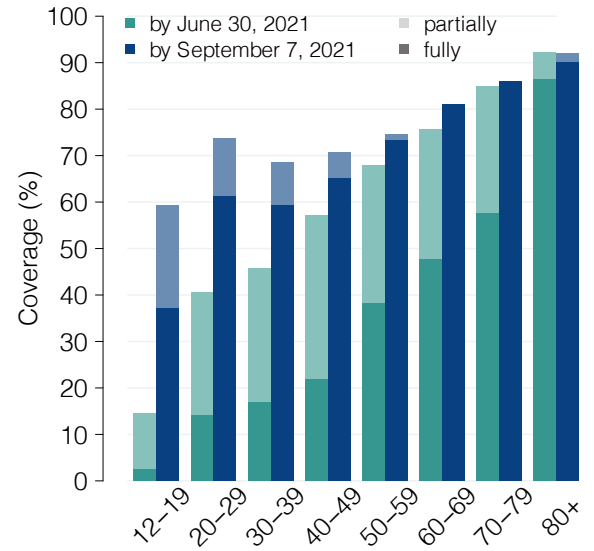

A.

Age group (years)

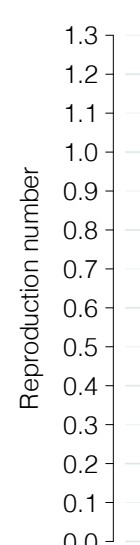

B. June 30, 2021
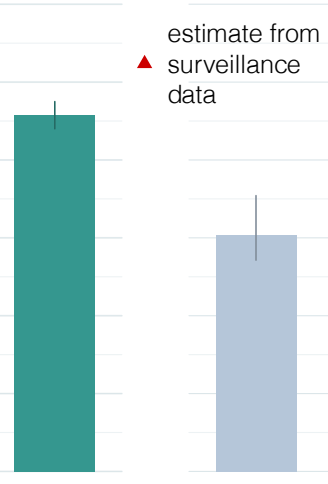

Alpha
September 7 , 2021

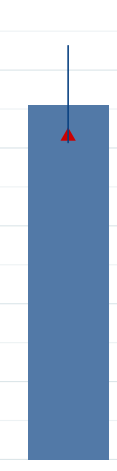

Alpha Delta

Figure 3. Vaccination coverage by June 30 and September 7, 2021, and effect of the replacement of the Alpha variant by the Delta variant. A) Comparison between the fraction of the Italian population that was partially and fully vaccinated by June 30, 2021, and by September 7, 2021, by age group. B) Green: net reproduction number estimated on June 30, 2021, when the Alpha variant was still largely dominant. Light blue: mean estimated value of the reproduction number, given the progression of the vaccination program until September 7 and under the assumption that the Alpha variant remained dominant. Dark blue: the same effect under the assumption of a $50 \%$ increase in transmissibility to reproduce the replacement of the Alpha variant with the Delta, occurred during the summer of 2021 [11-13, 25]. Vertical lines: $95 \% \mathrm{Cl}$. Red triangle: value of the reproduction number as estimated from surveillance data [29]; for this estimate, the $95 \% \mathrm{Cl}$ is not visible at the scale of the plot.

Future vaccination scenarios. We projected the potential impact of a further future progression of the vaccination campaign. To this aim, we evaluated scenarios in which all age groups will reach a given coverage $\Omega$; age groups which were already above that coverage on September 7, 2021, will remain unaffected (see the schematic example on Figure $4 \mathrm{~A}$ ). We then projected the reproduction number for different values of $\Omega$ and different proportions of pre-pandemic contacts that are resumed (Figure 4C). A complete return to the pre-pandemic lifestyle would still result in reproduction numbers significantly higher than the epidemic threshold of 1 and is therefore unlikely to achieve, even with an almost complete coverage of the population aged $12+$ years. This is due to the high transmissibility of the Delta variant and the imperfect protection against infection granted by vaccination. However, expanding the coverage would allow a significant resumption of social activity while maintaining the reproduction number under the epidemic threshold (Figure 4B), from a 56\% (95\% Cl: 45-62\%) of prepandemic contacts estimated for a coverage $>60 \%$ in all age classes (close to the uptake level already achieved on September 7,2021$)$ to a projected $76 \%(95 \% \mathrm{Cl}: 48-100 \%)$ for a $100 \%$ coverage of the eligible age groups ( 12 years or older). If a pediatric vaccine (for children aged 5 years and older) will be licensed and widely distributed, we projected that herd immunity may be reached even for a complete return to pre-pandemic social behavior with a coverage of at least $90 \%$ in all age classes (Figure 4D). Such herd immunity, however, would be only temporary, due to the waning vaccine protection over time.

We analyze the sensitivity of the estimated prospective reproduction numbers against different values for the increase in transmissibility of Delta compared to Alpha (and in absence of a pediatric vaccine). We show that for a transmissibility increase of $25 \%$, the proportion of pre-pandemic contacts that could be resumed without causing an epidemic would increase to $65-85 \%$, depending on the coverage scenario (Figure $5 \mathrm{~A}$ ). If Delta is $75 \%$ more transmissible than Alpha, the corresponding range would be limited to $45-60 \%$ (Figure 5B). 
medRxiv preprint doi: https://doi.org/10.1101/2021.03.19.21253893; this version posted November 15, 2021. The copyright holder for this preprint (which was not certified by peer review) is the author/funder, who has granted medRxiv a license to display the preprint in

It is made available under a CC-BY-NC-ND 4.0 International license .

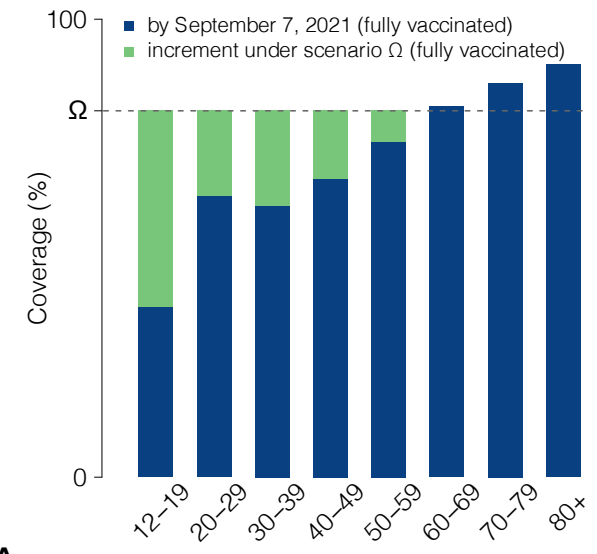

A.

Age group (years)

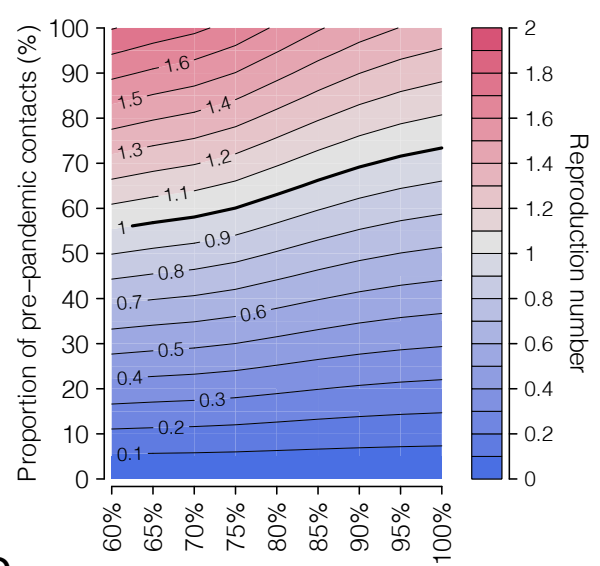

C.

Vaccination scenario $(\Omega)$
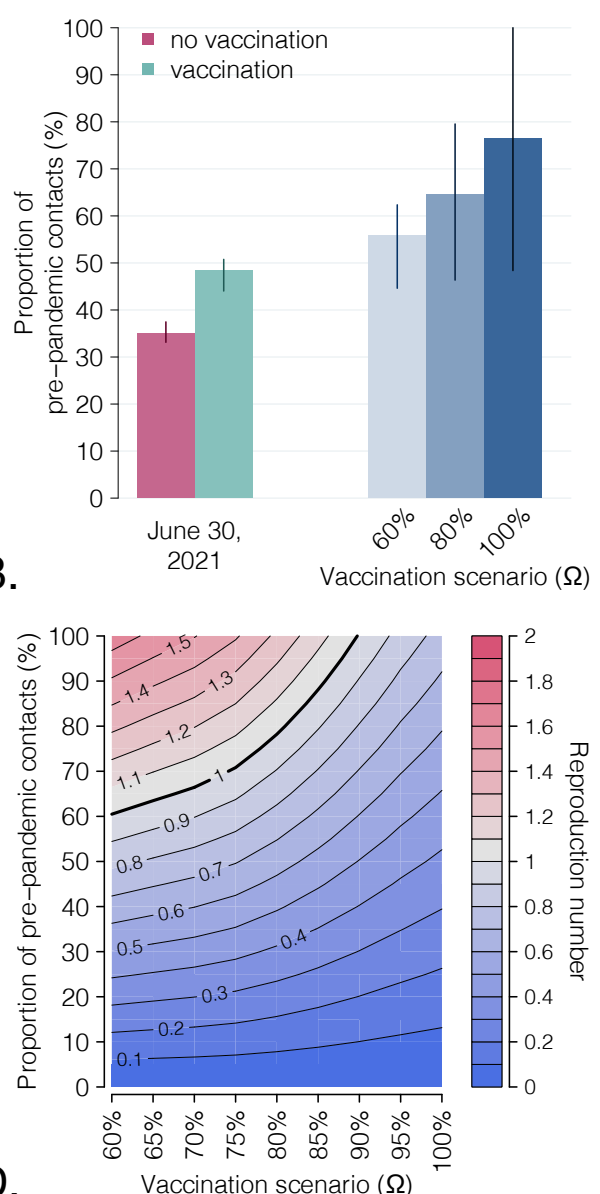

Figure 4. Scenarios for the expansion of vaccination coverage. A) Schematic of simulated scenarios. All age classes with coverage below a given value $\Omega$ are assumed to progress to $\Omega$; all age classes above $\Omega$ will remain at the coverage level achieved on Sep 7. B) Proportion of pre-pandemic contacts corresponding to a reproduction number of 1 for three selected vaccination scenarios; levels estimated to be active on June 30, 2021 (with and without vaccination) are reported for comparison. Bars: mean; vertical lines: $95 \% \mathrm{Cl}$. C) Heatmap of the estimated reproduction number for different vaccination scenarios ( $x$ axis) and different levels of social activity (y axis). Contour lines discriminate different values of the reproduction number. The thicker contour line represents the epidemic threshold of 1 . D) As C, but assuming that coverage $\Omega$ is achieved also in pediatric agegroups (5-11 years). 

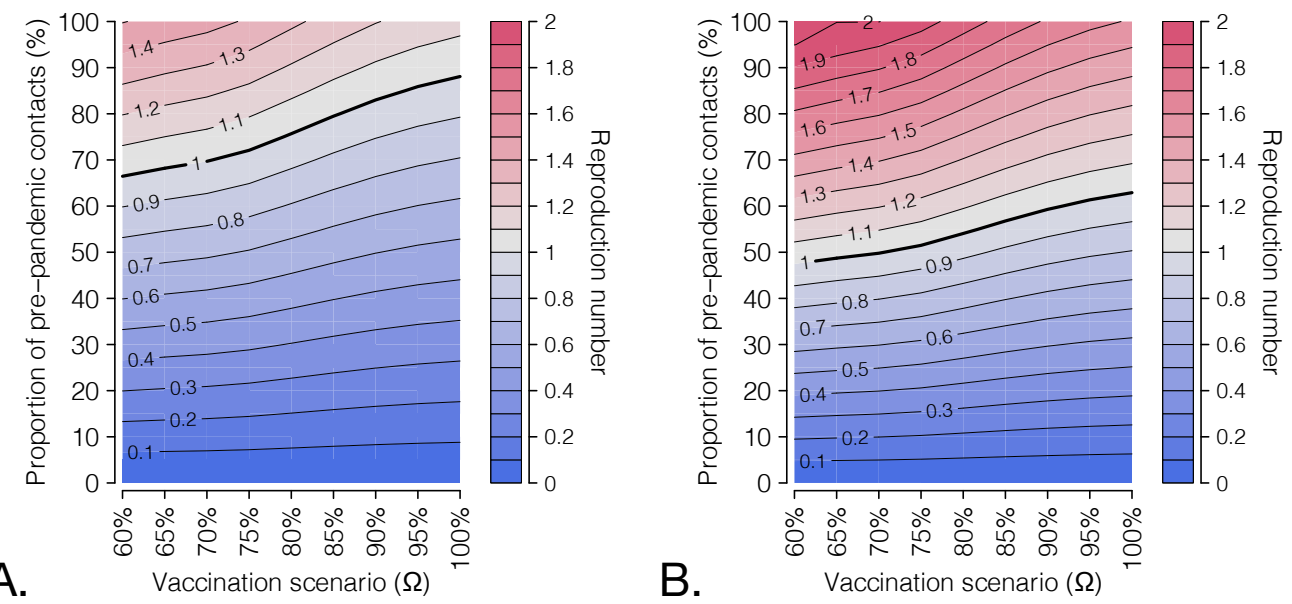

Figure 5. Sensitivity analysis with respect to the transmissibility increase of the Delta with respect to the Alpha variant. Heatmap of the projected reproduction number for different vaccination scenarios ( $x$ axis) and different levels of social activity (y axis), under a transmissibility increased by A) $25 \%$ and B) $75 \%$, compared to the Alpha variant.

\section{Discussion}

In this work, we quantified the retrospective and prospective impact of the COVID-19 vaccination campaign in Italy, which kicked off on December 27, 2020. First, we show that in the first half of 2021, a similar epidemic trajectory in absence of the vaccine would have resulted in a $27 \%(95 \% \mathrm{Cl} 15-47 \%)$ excess of COVID-19 deaths compared to the ones observed in the same period. This would have also required a reduction of social activity by one quarter (from $48 \%$ to $35 \%$ of pre-pandemic contacts at the end of June). In addition, a much higher risk for further waves of infection would be maintained, with an average effective reproduction number on June 30, 2021, of 2.6, instead of the 1.9 estimated in the presence of vaccination. Second, our results suggest that the replacement of the Alpha variant (and of other lineages) with the more transmissible Delta variant during the month of July was offset by the progression of the vaccination campaign in the months of July and August, resulting in a value of the reproduction number in early September that was similar to the one estimated at the end of June 2021. Summer vaccinations, however, did not reduce the transmissibility alone, but also the risk of severe disease and death in the population, given the high effectiveness of vaccines against these endpoints; thus, despite the Delta variant, the epidemiological outlook at the start of September 2021 was likely better than that at the end of June 2021. Finally, we estimate that expanding the vaccine coverage will allow a further increase of social activity while maintaining the reproduction number below the epidemic threshold. However, the high transmissibility of Delta and the imperfect vaccine protection against infection will not allow a complete return of society to the pre-pandemic life without the risk of occurrence of further pandemic waves. Should a pediatric vaccine (for ages 5 and older) be licensed and a coverage $>90 \%$ be achieved in all age classes, a complete return to prepandemic society could still be envisioned. For these estimates, we assume that between September 7, 2021, and the time the coverage for that vaccination scenario has been reached, the alteration of the population immunity profile due to the opposite forces of waning immunity and of the continued circulation of SARS-COV-2 will be negligible, compared to that caused by the progression of vaccination. This assumption may be broken if large waves of COVID-19 occur before reaching the considered coverage or if enough time elapses (several months) for a substantial waning of immunity. 
medRxiv preprint doi: https://doi.org/10.1101/2021.03.19.21253893; this version posted November 15,2021 . The copyright holder for this preprint (which was not certified by peer review) is the author/funder, who has granted medRxiv a license to display the preprint in

It is made available under a CC-BY-NC-ND 4.0 International license .

213 However, the administration of booster doses that is taking off in the fall of 2021 in countries with a

214 high population coverage, including Italy [30], will likely reduce the risks related to waning immunity.

215 One limitation of this study is that we implicitly assumed that vaccinated and unvaccinated individuals

216 have the same probability of contacting each other. However, it is known that vaccine hesitancy

217 clusters spatially and demographically [31], increasing the probability of local outbreaks in

218 undervaccinated pockets even when the average reproduction number is below the epidemic

219 threshold. To explore this effect, data on the clustering of COVID-19 vaccine hesitancy are warranted.

220 We did not consider the effect on our results of other features of the Delta variant, such as its

221 potential ability to escape natural immunity [24, 32-34], which is still partially undefined. In addition, even the increased transmissibility of Delta is subject to several unknowns; its value was estimated in situations where physical distancing restrictions were broadly active and thus a large proportion of interactions were with close contacts $[12,13]$. It is possible that, as interventions relax and social contacts increases, the estimated transmission advantage of Delta over Alpha (about 50\%) will be different. In a sensitivity analysis, we showed that the actual value of this parameter critically affects epidemiological prospects.

The dynamics of loss of protection over time for different population demographics (age, comorbidities) and clinical endpoints (infection, death, transmissibility of breakthrough infections) will likely affect future COVID-19 trajectories and must be better elucidated with long-term follow-up studies. Based on preliminary studies [22, 23], we assumed an average duration of two years for the protection conferred by both infection and vaccination, and equal for all individuals. In sensitivity analyses, we show that different durations of the natural immunity may affect our estimates of the effective reproduction number at the end of June, 2021 and therefore impact prospective scenarios for the next year (see Appendix). Similarly, if breakthrough infections were as transmissible as infections in unvaccinated individual, this would increase the estimated reproduction numbers and reduce the levels of societal reopening that would be affordable (see Appendix).

This work highlights the multiple epidemiological and social benefits allowed by the vaccination efforts in terms of averted deaths, reopening of social activity, and reduced risks of further epidemic waves. In addition, our study shows the potential for further resuming social activities granted by the expansion of vaccination coverage in the perspective of "living with the virus". In particular, the availability of pediatric vaccines, which, as of October 2021, are under scientific investigation and regulatory scrutiny [35], could greatly contribute to societal reopening should the coverage be sufficiently high. However, the acceptability of a pediatric vaccine may be limited by the perceived small risk of COVID-19 disease in children, especially if adverse vaccine events will be recorded even with very low rates [36].

The scenario of complete resumption of pre-pandemic social life would entail removing all of the persisting factors that today still reduce the number of an individual's contacts compared to the preCOVID-19 era. These include: residual governmental limitations (e.g., capacities in stadiums and discotheques, number of people who can be seated together at restaurants indoors, etc.); organizational measures reducing crowding (e.g., capacity of workplace spaces and the use of work from home, distancing of desks in schools, mandatory booking for recreational and cultural activities, regulations for weddings and other large events); social distancing etiquette; and individual choices to reduce one's own risks of infection. In addition, several preventive measures further reduce the contacts that are important for transmission (those considered in the model) without significantly 
medRxiv preprint doi: https://doi.org/10.1101/2021.03.19.21253893; this version posted November 15,2021 . The copyright holder for this preprint (which was not certified by peer review) is the author/funder, who has granted medRxiv a license to display the preprint in

It is made available under a CC-BY-NC-ND 4.0 International license .

256

257

258

259

260

261

262

263

264

265

266

267

268

269

270

271

272

273

274

275

276

277

278

279

280

281

282

283

284

285

286

287

288

289

290

291

292

293

294

295

296

297

298

affecting social interactions, e.g., mandates for EU digital COVID-19 certificate [37] (currently required in Italy for accessing workplaces, schools, and indoor recreational facilities), ventilation policies and air filtering systems on public transport, plexiglass separators between restaurant tables or at counters of commercial and public offices, face masks, and testing, tracing and isolation protocols. Although quantifying the impact of each of these measures and norms is extremely hard, it is likely that many of them will linger for a long time without a significant negative influence on either the economy or the social life of individuals. Therefore, a complete resumption of pre-pandemic contacts in the sense considered by the model may not necessarily be a key objective. Depending on the measures that will be maintained and on the acquired coverage, we estimate the SARS-CoV-2 reproduction number to take values between 0.7 (if contacts will not increase and coverage will be close to 100\%) and 1.8 (if social activity will be fully resumed and norms will be abandoned without increases in vaccine coverage).

Finally, we stress that our prospective results need to be revised in case of the future emergence of new hypertransmissible variants. Such a possibility could jeopardize the gains afforded by vaccination programs, forcing new setbacks in the recovery of social contacts and exacerbating the burden of a potential further epidemic resurgence.

\section{Methods}

We developed an age-structured stochastic model of SARS-CoV-2 transmission and vaccination, based on a susceptible-infectious-removed-susceptible (SIRS) scheme $[17,18]$. The population is stratified by age (17 5-year age groups from 0 to 84 years plus one age group for individuals aged 85 years or older) and presence/absence of comorbidities. Mixing patterns are encoded by an age-specific social contact matrix estimated prior to the COVID-19 pandemic [20]. Susceptibility to SARS-CoV-2 infection is assumed to be age-dependent (lower in children under 15 years of age and higher for the elderly above 65 years, compared to individuals aged 15-65) [38]. Infectiousness was assumed to be homogeneous by age groups and symptomatic status [38, 39]. We consider a basic reproduction number $R_{0}$ for historical lineages of $3.0[19,40,41]$. The model was used to simulate the vaccination campaign and the evolution of COVID-19 epidemiology in Italy between December 27, 2020 (start of vaccination) and June 30, 2021. Throughout this period, the dominant variant was Alpha [24]; therefore, in our retrospective investigation we considered an increase in transmissibility by $50 \%$ compared to historical lineages [24, 42, 43].

The rollout of the vaccination campaign is modeled using detailed data on the daily age-specific number of doses administered over the considered period [21]. Individuals are considered eligible for vaccination, independently of a previous diagnosis of SARS-CoV-2 infection. To account for preferential administration of different types of vaccines by age group, we estimated the age-specific vaccine efficacy against infection by weighting the efficacy of a specific vaccine type (mRNA vs. viral vectors) by the number of vaccines of that type administered to each age group [21], considering a vaccine efficacy against infection of $89 \%$ after two doses of mRNA vaccine, and of $62 \%$ after two doses of viral vector vaccine $[8,9]$. The efficacy against death was set to $80.6 \%$ in partially and $96.4 \%$ in fully vaccinated individuals [28]. Breakthrough infections (i.e., infections in vaccinated individuals) were assumed to be half as infectious as those in unvaccinated individuals [44, 45]; we additionally considered a sensitivity analysis where the infectiousness is the same. Immune protection is assumed to wane after an exponentially distributed time (average 2 years $[22,23]$ in the baseline for both the 
medRxiv preprint doi: https://doi.org/10.1101/2021.03.19.21253893; this version posted November 15,2021 . The copyright holder for this preprint (which was not certified by peer review) is the author/funder, who has granted medRxiv a license to display the preprint in

It is made available under a CC-BY-NC-ND 4.0 International license .

299

300

301

302

303

304

305

306

307

308

309

310

311

312

313

314

315

316

317

318

319

320

321

322

323

324

325

326

327

328

329

330

331

332

333

334

335

336

337

338

339

340

341

342

343

344

345

natural and vaccine-induced protection; alternative values are considered as sensitivity analyses). After waning of protection, individuals are considered fully susceptible.

To reproduce the epidemic curve over the study period, we adjusted a scaling factor representing the proportion of pre-pandemic contacts that were active on a given day, in such a way that the model's reproduction number (estimated via the Next Generation Matrix approach [46, 47]) would match the corresponding estimate from surveillance data [10]. We compared estimates obtained with the actual vaccination rollout against those that would be required in a hypothetical scenario without vaccination to obtain the same epidemic curve. We evaluated the prospective impact of the vaccination campaign by considering the replacement of the Alpha with the Delta variant (occurred in July 2021) [25], which is assumed to be 50\% more transmissible [11-13] (transmissibility increases of $25 \%$ and $75 \%$ are considered in sensitivity analyses). We also considered vaccination scenarios where the age-specific vaccination coverage achieved by September 7, 2021, is incremented for all age classes that were below a given target coverage $\Omega$ at that date and left unchanged for age classes above. For each scenario, we estimate reproduction numbers under different degrees of resumption of pre-pandemic contacts (from 0\% to 100\%). Full model details are reported in Appendix.

\section{Competing interest and funding}

M.A. has received research funding from Seqirus. The funding is not related to COVID-19. All other authors declare no competing interest. V.M., G.G., F.R., P.Po., F.T., M.M., P. Pe. and S.M. acknowledge funding from EU grant 874850 MOOD (catalogued as MOOD 020). V.M., G.G., P.Po., and S.M. acknowledge funding from the VRT Foundation Trento project COVIDVAX. The contents of this publication are the sole responsibility of the authors and don't necessarily reflect the views of the funders.

\section{References}

1. WHO coronavirus (COVID-19) dashboard. https://covid19.who.int/. Accessed on Mar 15, 2021.

2. Trentini, F. et al. The pressure on healthcare system and intensive care utilization during the COVID-19 outbreak in the Lombardy region: a retrospective observational study on 43,538 hospitalized patients. Am. J. Epidemiol. doi: 10.1093/aje/kwab252

3. McKibbin, W. \& Fernando, R. The economic impact of COVID-19. Economics in the Time of COVID-19, 45 (2020).

4. Pfefferbaum, B. \& North, C. S. Mental health and the Covid-19 pandemic. N. Engl. J. Med., 383(6), 510512 (2020).

5. Our world in data. COVID-19 Data Explorer. https://ourworldindata.org/explorers/coronavirus-dataexplorer. Accessed on Oct 15, 2021.

6. Shapiro, J., Dean, N. E., Madewell, Z. J., Yang, Y., Halloran, M. E., Longini, I. Efficacy Estimates for Various COVID-19 Vaccines: What we Know from the Literature and Reports. medRxiv. doi: https://doi.org/10.1101/2021.05.20.21257461 (2021).

7. Haas, E. J. et al. Impact and effectiveness of mRNA BNT162b2 vaccine against SARS-CoV-2 infections and COVID-19 cases, hospitalisations, and deaths following a nationwide vaccination campaign in Israel: an observational study using national surveillance data. Lancet, 397.10287: 1819-1829 (2021).

8. Emary, K. R. W. et al. Efficacy of ChAdOx1 nCoV-19 (AZD1222) vaccine against SARS-CoV-2 variant of concern 202012/01 (B.1.1.7): an exploratory analysis of a randomised controlled trial. Lancet, 397(10282), 1351-1362 (2021).

9. Abu-Raddad, L. J., Chemaitelly, H., Butt, A. A. \&s National Study Group for COVID-19 Vaccination. Effectiveness of the BNT162b2 Covid-19 Vaccine against the B.1.1.7 and B.1.351 Variants. N. Engl. J. Med., 385(2):187-189. doi: 10.1056/NEJMc2104974 (2021). 
medRxiv preprint doi: https://doi.org/10.1101/2021.03.19.21253893; this version posted November 15,2021 . The copyright holder for this preprint (which was not certified by peer review) is the author/funder, who has granted medRxiv a license to display the preprint in It is made available under a CC-BY-NC-ND 4.0 International license .

10. Istituto Superiore di Sanità. Epidemia COVID-19 - Aggiornamento nazionale 6 ottobre 2021. Available at: https://www.epicentro.iss.it/coronavirus/bollettino/Bollettino-sorveglianza-integrata-COVID-19 6ottobre-2021.pdf

11. Campbell, F. et al. Increased transmissibility and global spread of SARS-CoV-2 variants of concern as at June 2021. Eurosurveillance 26(24):2100509. doi: 10.2807/1560-7917.ES.2021.26.24.2100509 (2021).

12. Keeling, M. J. Estimating the Transmission Advantage for B.1.617.2. Available at: https://assets.publishing.service.gov.uk/government/uploads/system/uploads/attachment data/file/9 93156/S1269 WARWICKTransmission Advantage.pdf

13. Alizon, S. et al. Rapid spread of the SARS-CoV-2 Delta variant in some French regions, June 2021. Eurosurveillance, 26(28): 2100573 (2021).

14. Kofman, A., Kantor, R. \& Adashi, E.Y. Potential COVID-19 Endgame Scenarios: Eradication, Elimination, Cohabitation, or Conflagration? JAMA, 326(4):303-304. doi:10.1001/jama.2021.11042 (2021).

15. Baker, M. G., Wilson, N. \& Blakely, T. Elimination could be the optimal response strategy for COVID-19 and other emerging pandemic diseases. BMJ 371, m4907 (2020).

16. Piot, P., \& Spencer, J. Towards Societies Living with COVID-19 China CDC Weekly, 3(7): 144-145 (2021). doi: $10.46234 /$ ccdcw2021.041

17. Marziano, V. et al. Retrospective analysis of the Italian exit strategy from COVID-19 lockdown. Proc. Natl Acad. Sci. 118, e2019617118 (2021).

18. Yang, J. et al. Despite vaccination, China needs non-pharmaceutical interventions to prevent widespread outbreaks of COVID-19 in 2021. Nat. Hum. Behav. 5, 1009-1020 (2021). https://doi.org/10.1038/s41562-021-01155-z

19. Riccardo, F. et al. Epidemiological characteristics of COVID-19 cases and estimates of the reproductive numbers 1 month into the epidemic, Italy, 28 January to 31 March 2020. Eurosurveillance 25, 2000790 (2020).

20. Mossong, J. et al. Social contacts and mixing patterns relevant to the spread of infectious diseases. PLoS Med. 5, e74 (2008).

21. Struttura Commissariale per l'Emergenza Covid-19. Open data on COVID-19 vaccination in Italy. https://github.com/italia/covid19-opendata-vaccini. Accessed on October 15, 2021

22. Hall, V. J. et al. SARS-CoV-2 infection rates of antibody-positive compared with antibody-negative health-care workers in England: a large, multicentre, prospective cohort study (SIREN). Lancet 397, 1459-1469 (2021).

23. Andrews, N. Vaccine effectiveness and duration of protection of Comirnaty, Vaxzevria and Spikevax against mild and severe COVID-19 in the UK. medRxiv. doi: https://doi.org/10.1101/2021.09.15.21263583 (2021).

24. Stefanelli P. et al. Co-circulation of SARS-CoV-2 Alpha and Gamma variants in Italy, February-March 2021. Eurosurveillance (in press).

25. Istituto Superiore di Sanità. Prevalenza e distribuzione delle varianti di SARS-CoV-2 di interesse per la sanità pubblica in Italia - Rapporto n. 5 del 23 luglio 2021. Available at: https://www.epicentro.iss.it/coronavirus/pdf/sars-cov-2-monitoraggio-varianti-rapporti-periodici-23luglio-2021.pdf

26. Istituto Superiore di Sanità. Prevalenza e distribuzione delle varianti di SARS-CoV-2 di interesse per la sanità pubblica in Italia - Rapporto n. 9 del 17 settembre 2021. Available at: https://www.epicentro.iss.it/coronavirus/pdf/sars-cov-2-monitoraggio-varianti-rapporti-periodici-17settembre-2021.pdf

27. Istituto Superiore di Sanità. COVID-19 ISS open data - EpiCentro. Available at: https://www.epicentro.iss.it/coronavirus/open-data/covid 19-iss.xIsx. Accessed on October 15, 2021

28. Istituto Superiore di Sanità. Epidemia COVID-19 - Aggiornamento nazionale 28 luglio 2021. Available at: https://www.epicentro.iss.it/coronavirus/bollettino/Bollettino-sorveglianza-integrata-COVID-19 28luglio-2021.pdf 
medRxiv preprint doi: https://doi.org/10.1101/2021.03.19.21253893; this version posted November 15,2021 . The copyright holder for this preprint (which was not certified by peer review) is the author/funder, who has granted medRxiv a license to display the preprint in It is made available under a CC-BY-NC-ND 4.0 International license .

29. Istituto Superiore di Sanità. Epidemia COVID-19 - Aggiornamento nazionale 22 settembre 2021. Available at: https://www.epicentro.iss.it/coronavirus/bollettino/Bollettino-sorveglianza-integrataCOVID-19 22-settembre-2021.pdf

30. Ministero della Salute. Avvio della somministrazione di dosi "booster" nell'ambito della campagna di vaccinazione anti SARS-CoV-2/COVID-19. Available at: trovanorme.salute.gov.it/norme/renderNormsanPdf?anno=2021\&codLeg $=82953 \&$ parte $=1 \% 20 \&$ serie $=$ null

31. Salathé, M. \& Bonhoeffer, S. The Effect of Opinion Clustering on Disease Outbreaks. J. R. Soc. Interface 5, 1505-1508 (2008).

32. Faria, N. R. et al. Genomics and epidemiology of the P.1 SARS-CoV-2 lineage in Manaus, Brazil. Science 372, 815-821 (2021).

33. Greaney, A. J. et al. Comprehensive mapping of mutations in the SARS-CoV-2 receptor-binding domain that affect recognition by polyclonal human plasma antibodies. Cell Host Microbe 29, 463-476 (2021).

34. Wang, P. et al. Antibody resistance of SARS-CoV-2 variants B.1.351 and B.1.1.7. Nature 593, 130-135 (2021).

35. Pfizer Inc. Pfizer and BioNtech submit initial data to U.S. FDA from pivotal trial of COVID-19 vaccine in children 5 to $<12$ years of age. Available at: https://www.pfizer.com/news/press-release/press-releasedetail/pfizer-and-biontech-submit-initial-data-us-fda-pivotal

36. Bozkurt, B., Kamat, I., \& Hotez, P. J. Myocarditis with COVID-19 mRNA vaccines. Circulation, 144(6), 471-484 (2021).

37. European Commission. EU Digital COVID Certificate. https://ec.europa.eu/info/live-work-traveleu/coronavirus-response/safe-covid-19-vaccines-europeans/eu-digital-covid-certificate en

38. Hu, S. et al. Infectivity, susceptibility, and risk factors associated with SARS-CoV-2 transmission under intensive contact tracing in Hunan, China. Nat. Commun. 12, 1533 (2021).

39. Sun, K. et al. Transmission heterogeneities, kinetics, and controllability of SARS-CoV-2. Science 371, eabe2424 (2021).

40. Cereda, D. et al. The early phase of the COVID-19 outbreak in Lombardy, Italy. https://arxiv.org/pdf/2003.09320.pdf (2020).

41. Guzzetta, G. et al. Impact of a nationwide lockdown on sars-cov-2 transmissibility, Italy. Emerg. Infect. Dis. 27, 267 (2021).

42. Volz, E. et al. Assessing transmissibility of SARS-CoV-2 lineage B.1.1.7 in England. Nature 593, 266-269 (2021).

43. Davies, N. G. et al. Estimated transmissibility and impact of SARS-CoV-2 lineage B.1.1.7 in England. Science https://doi.org/10.1126/science.abg3055 (2021).

44. Harris, R.J., Hall, J.A., Zaidi, A., Andrews, N.J., Dunbar, J. K., Dabrera, G. Effect of Vaccination on Household Transmission of SARS-CoV-2 in England. N. Engl. J. Med. 385(8):759-760. doi: 10.1056/NEJMc2107717 (2021).

45. Lipsitch, M. \& Kahn, R. Interpreting vaccine efficacy trial results for infection and transmission. Vaccine, 39(30): 4082-4088 (2021).

46. Diekmann, O., Heesterbeek, J. A. P. \& Metz, J. A. On the definition and the computation of the basic reproduction ratio $\mathrm{RO}$ in models for infectious diseases in heterogeneous populations. J. Math Biol. 28, 365-382 (1990).

47. Diekmann, O., Heesterbeek, J. A. \& Roberts, M. G. The construction of next-generation matrices for compartmental epidemic models. J. R. Soc. Interface 7, 873-885 (2010). 